\title{
Association of the interleukin 1 beta gene and brain spontaneous activity in amnestic mild cognitive impairment
}

\author{
Liying Zhuang ${ }^{1,2}$, Xiaoyan Liu ${ }^{1,2}$, Xiaohui Xu ${ }^{1,2}$, Chunxian Yue ${ }^{1,2}$, Hao Shu ${ }^{1,2}$, Feng Bai ${ }^{1,2}$, Hui Yu ${ }^{1}$, \\ Yongmei Shi ${ }^{1,2}$ and Zhijun Zhang ${ }^{1,2^{*}}$
}

\begin{abstract}
Purpose: The inflammatory response has been associated with the pathogenesis of Alzheimer's disease (AD). The purpose of this study is to determine whether the rs 1143627 polymorphism of the interleukin-1 beta (IL-1 $\beta$ ) gene moderates functional magnetic resonance imaging (fMRI)-measured brain regional activity in amnestic mild cognitive impairment (aMCl).

Methods: Eighty older participants (47 with $\mathrm{aMCl}$ and 33 healthy controls) were recruited for this study. All of the participants were genotyped for variant rs 1143627 in the ILIB gene and were scanned using resting-state fMRI. Brain activity was assessed by amplitude of low-frequency fluctuation (ALFF).

Results: aMCI patients had abnormal ALFF in many brain regions, including decreases in the inferior frontal gyrus, the superior temporal lobe and the middle temporal lobe, and increases in the occipital cortex (calcarine), parietal cortex (Pcu) and cerebellar cortex. The regions associated with an interaction of group $X$ genotypes of rs1143627 C/T were the parietal cortex (left Pcu), frontal cortex (left superior, middle, and medial gyrus, right anterior cingulum), occipital cortex (left middle lobe, left cuneus) and the bilateral posterior lobes of the cerebellum. Regarding the behavioral significance, there were significant correlations between ALFF in different regions of the brain and with the cognitive scores of each genotype group.

Conclusions: The present study provided evidence that aMCI patients had abnormal ALFF in many brain regions. Specifically, the rs $1143627 \mathrm{C} / \mathrm{T}$ polymorphism of the IL1B gene may modulate regional spontaneous brain activity in aMCl patients.
\end{abstract}

Keywords: Amnestic mild cognitive impairment, Functional magnetic resonance imaging, Amplitude of low-frequency fluctuation, Interleukin-1 beta, Cognition

\section{Background}

Alzheimer's disease (AD) is the most common form of dementia worldwide. It is characterized by two microscopic neuropathologic hallmarks - senile plaques composed of amyloid beta $(A \beta)$ and neurofibrillary tangles composed of hyperphosphorylated tau. Mild cognitive impairment (MCI) is considered as an intermediate clinical state between normal aging and dementia [1].

\footnotetext{
* Correspondence: zhijunzhang@seu.edu.cn

${ }^{1}$ Medical School of Southeast University, 87 Dingjiaqiao Road, Nanjing, Jiangsu 210009, China

2Department of Neurology, Affiliated Zhongda Hospital of Southeast University, and the Institute of Neuropsychiatry of Southeast University, 87 Dingjiaqiao Road, Nanjing, Jiangsu 210009, China
}

According to the impaired domains of cognitive function, MCI can be divided into amnestic mild cognitive impairment (aMCI) and nonamnestic MCI [1]. aMCI is commonly regarded as the prodromal phase of $\mathrm{AD}$, with 10 times the annual conversion rate of normal aging [2].

As research of reliable biomarkers of $\mathrm{AD}$ advances, brain $A \beta$-plaque deposition and neurodegeneration have been labeled as the biomarker categories in $\mathrm{AD}$ [3]. However, the use of a more extensive biomarker profile, rather than any single measure, may help improve the sensitivity and selectivity of predicting AD earlier [4]. In addition to senile plaques and neurofibrillary tangles, the Neuroinflammation Working Group has reached a 
consensus that inflammation is involved in the pathogenesis of $\mathrm{AD}$ [5]. Biomarkers, such as cytokines, have already been assessed [6,7]. The proinflammatory cytokine interleukin-1 beta (IL-1 $\beta$ ) is a mental component in the inflammatory pathway and is overexpressed in the brain of $\mathrm{AD}$ patients. The gene for IL1B maps to chromosome 2q14. Several polymorphisms of this gene have been recognized [8]. Among these polymorphisms, the $-31 \mathrm{~T}>\mathrm{C}$ (rs1143627) polymorphism in the promoter region affects $I L 1 B$ gene expression particularly.

Functional neuroimaging techniques, which have been utilized in the study of AD/MCI, mainly include singlephoton emission computed tomography (SPECT), positron emission tomography (PET) and blood oxygenation level-dependent (BOLD) functional magnetic resonance imaging (fMRI). Resting-state fMRI has been developed as a new branch of this field. Compared with SPECT/ PET, resting-state fMRI has an advantage in that there is no radiation exposure. Biswal and colleagues have demonstrated that spontaneous low-frequency (typically 0.01 to $0.08 \mathrm{~Hz}$ ) fluctuations of the human brain, measured by resting state fMRI, are physiologically meaningful [9]. An approach to measure the amplitude of lowfrequency fluctuation (ALFF) of BOLD signals in early $\mathrm{AD}$ has been developed to explore regional neural function by Zang and colleagues [10]. To date, ALFF has been widely applied to the study of different brain disorders, including epilepsy [11], schizophrenia [12,13], drug addiction [14], posttraumatic stress disorder (PTSD) [15], attention deficit hyperactivity disorder (ADHD) $[16,17]$, multiple sclerosis (MS) [18], and aMCI [19]. This study aimed to determine whether rs1143627 moderates fMRI-measured brain regional activity in aMCI.

\section{Methods}

\section{Study participants}

A total of 47 aMCI patients and 33 healthy controls were recruited from the Affiliated Zhongda Hospital of Southeast University and communities in Nanjing, China. All of the participants, who were Han Chinese and right-handed, were interviewed by two trained senior neurologists. This study was approved by the Southeast University Ethics Committee and informed consent was obtained from all of the participants.

A diagnosis of aMCI, including single domain (the impairment involves only the memory domain) and multiple domain (impairment in the memory domain plus impairments in at least one other cognitive domain), was made following the recommendations of Petersen [20] and others [21], including (1) a subjective memory complaint, preferably corroborated by an informant, (2) an objective memory impairment, such as a score of less than or equal to 1.5 SD of age-adjusted and educationadjusted norms on the 20-min delayed recall of auditory verbal learning test (AVLT) (the cutoff was $\leq 4$ correct responses on 12 items for $\geq 8$ years of education), (3) normal general cognitive functioning, measured by a mini-mental state examination (MMSE) score of 24 or higher, (4) a clinical dementia rating scale (CDR) of 0.5 , with at least a 0.5 in the memory domain, (5) normal or minimal impairment in the activities of daily living (ADL), a score of 20 to 26, and (6) absence of dementia, or not sufficient to meet the National Institute of Neurological and Communicative Disorders and Stroke and the Alzheimer's Disease and Related Disorders Association (NINCDS-ADRDA) criteria for AD. Participants were excluded if they had a past history of known stroke (modified Hachinski score $>4$ ), alcoholism, head trauma, Parkinson's disease, epilepsy, major depression (excluded by the self-rating depression scale) or any other neurological or psychiatric illness (excluded by clinical assessment and case history), major medical illness (for example, cancer, anemia, thyroid dysfunction), or severe visual or hearing loss.

Control participants were required to have a CDR of 0 , a MMSE score $\geq 26$, and a delayed recall score $>4$ for those with 8 or more years of education.

\section{DNA isolation and SNP genotyping}

Four milliliters of peripheral blood from each participant was collected into EDTA-containing vacutainer tubes and stored at $-80^{\circ} \mathrm{C}$. Genomic DNA was isolated using the Wizard Genomic DNA purification Kit (Promega, Madison, WI, USA) according to the manufacturer's protocol. The PCR primers were designed using Genotyping Tools (Sequenom, Hamburg, Germany) and MassARRAY Assay Design software (Sequenom) and were synthesized by Invitrogen China (Beijing, China). PCR amplification reactions $(5 \mu \mathrm{l})$ were performed in standard 384-well plates using $10 \mathrm{ng}$ genomic DNA, $0.5 \mathrm{U}$ of Taq polymerase (HotStarTaq, Qiagen, Valencia, CA, USA), $0.1 \mu \mathrm{l}$ of $25 \mathrm{mM}$ each dNTP and $0.5 \mathrm{pmol}$ of each PCR primer. PCR thermal cycling was carried out for 4 min at $94^{\circ} \mathrm{C}$, followed by 45 cycles of $20 \mathrm{~s}$ at $94^{\circ} \mathrm{C}, 30 \mathrm{~s}$ at $56^{\circ} \mathrm{C}, 1 \mathrm{~min}$ at $72^{\circ} \mathrm{C}$ and then $3 \mathrm{~min}$ at $72^{\circ} \mathrm{C}$. Next, 0.5 $\mathrm{U}$ of shrimp alkaline phosphatase (SAP; Sequenom) and $0.17 \mu \mathrm{l}$ of buffer were added to the PCR reaction products and were incubated for $20 \mathrm{~min}$ at $37^{\circ} \mathrm{C}$, then inactivated for $5 \mathrm{~min}$ at $85^{\circ} \mathrm{C}$ to get rid of free dNTP. PostPCR reactions, also called single base extension (SBE) reactions, which were performed in a final volume of 9 $\mu \mathrm{l}$ containing $0.804 \mu \mathrm{l}$ of each Primer Mix, $0.041 \mu \mathrm{l}$ of iPLEX enzyme, and $0.2 \mu \mathrm{l}$ of Terminator, and consisted of 40 cycles of denaturation at $94^{\circ} \mathrm{C}$ and a final $3 \mathrm{~min}$ extension step at $72^{\circ} \mathrm{C}$. The products of the iPLEX reaction were purified by $6 \mathrm{mg}$ Clean Resin (Sequenom) and were then spotted on a SpectroChip (Sequenom). Data 
were processed and analyzed by MassARRAY TYPER 4.0 software (Sequenom).

\section{MRI scanning}

All of the participants were scanned with a General Electric 1.5 Tesla scanner (General Electric Medical Systems, Miwaukee, WI, USA) by two experienced doctors from the radiology department following a standard imaging protocol. The participants lay supine with their heads snugly fixed by a belt and pads were used to minimize head motion. Functional images (T2* weighted images) were obtained using a GRE-EPI pulse sequence with the following parameters: 30 contiguous axial slices, slice thickness $/$ gap $=4.0 / 0 \mathrm{~mm}$, in-plane resolution $=3.75 \times$ $3.75 \mathrm{~mm}^{2}, \mathrm{TR}=3000 \mathrm{~ms}, \mathrm{TE}=40 \mathrm{~ms}$, flip angle $=90^{\circ}$, acquisition matrix $=64 \times 64, \mathrm{FOV}=240 \times 240 \mathrm{~mm}$. This acquisition sequence generated 142 volumes in 7 min and $6 \mathrm{~s}$. In addition, three-dimensional T1-weighted axial images covering the whole brain were obtained using a spoiled gradient echo (SPGR) sequence (TR = $9.9 \mathrm{~ms}, \mathrm{TE}=2.1 \mathrm{~ms}$, slice thickness $=2.0 \mathrm{~mm}$, gap $=0$ $\mathrm{mm}$, flip angle $=15^{\circ}, \mathrm{FOV}=240 \times 240 \mathrm{~mm}$, acquisition matrix $=256 \times 192)$. Participants were instructed to keep their eyes closed, bodies aplanatic and not to think systematically or fall asleep during the scanning.

\section{MRI data preprocessing}

The first eight volumes of each functional time course were discarded to allow for T1 equilibrium and to allow the participants to adapt to the scanning conditions. Slice timing, head motion correction, and spatial normalization were conducted using SPM5 (http://www. fil.ion.ucl.ac.uk/spm). Participants with head motion of more than a $3.0 \mathrm{~mm}$ maximum displacement in any direction $(\mathrm{x}, \mathrm{y}$, and $\mathrm{z})$ or $3.0^{\circ}$ of angular motion throughout the course of the scan were excluded from this study. The resulting images were spatially smoothed (full width at half maximum (FWHM) $=8 \mathrm{~mm}$ ) using SPM 5. Then, REST software (http://resting-fmri.sourceforge.net) was used for linear trend removal temporal band-pass filtering $(0.01 \sim 0.08 \mathrm{~Hz})[9,22]$.

\section{ALFF calculation}

ALFF was calculated using REST software similar to that used in previous studies $[17,23,24]$. Briefly, for a given voxel, after image preprocessing, the time series of resultant images was first converted to the frequency domain using a Fast Fourier Transform and the power spectrum was then acquired. The square root was computed at each frequency of the power spectrum and averaged between 0.01 and $0.08 \mathrm{~Hz}$, and this averaged square root was termed ALFF. For standardization, a whole-brain mask was created by removing the background and other tissues outside the brain in the anatomical images using MRIcro software (http://www. mricro.com) [25-27].

\section{Voxelwise-based gray matter volume correction}

To control for possible differences in ALFF that may be explained by differences in gray matter distribution between participants and to isolate the functional changes in components that cannot be attributed to anatomical differences, and thus are likely due to genuine functional differences, our study adopted estimates of a voxel's likelihood of containing gray matter as a covariate (nuisance variable) in the analysis of the resting-state functional data [28]. First, voxel-based morphometry (VBM) $[29,30]$ was used to explore gray matter volume maps of each participant. Second, the maps were transformed into the same standard space as the resting-state fMRI images using affine linear registration [31]. Finally, these resulting voxelwise gray matter volume maps were input as covariates in the analysis of the functional data. Voxelwise-based gray matter volume correction was used for each participant. The ALFF corrected by the voxelwise-based gray matter volume was then analyzed as follows.

\section{Statistical analysis}

To determine the effects of group and genotype on ALFF, we performed a two-way analysis of variance (ANOVA) on a voxel-by-voxel basis with groups (aMCI patients and healthy controls) and genotypes (CC, CT and TT). To further explore the details of those clusters showing significant main effects and interactions, post hoc $t$ tests were performed. All the statistical maps were corrected for multiple comparisons using a significance level of $P$ value $<0.05$, based on Monte Carlo simulations (parameters: single voxel $P$ value $=0.05$, a minimum cluster size of $10503 \mathrm{~mm}^{3}$, FWHM $=8 \mathrm{~mm}$, with mask. See program AlphaSim by D. Ward, and http://afni. nimh.nih.gov/pub/dist/doc/manual/AlphaSim.pdf). Finally, we performed a correlative analysis between the neuropsychological test scores and the ALFF values of the clusters showing significant interactions between group and genotype $(P<0.05)$.

\section{Results}

\section{Neuropsychological data}

The demographic and neuropsychological data for all the participants are shown in Table 1 . There were no significant differences in gender, age, education levels between the two groups. Compared with the controls, the aMCI participants showed deficits in memory (auditory verbal learning test delayed recall and ReyOsterrieth complex figure test delayed recall), construction (clock drawing test), attention/psychomotor speed (trail making test A, symbol digit modalities and digit 
Table 1 Demographic and neuropsychological data between aMCl group and healthy control group

\begin{tabular}{|c|c|c|c|}
\hline Items & $\operatorname{aMCl} \operatorname{group}(n=47)$ & Control group $(n=33)$ & $P$ value \\
\hline Gender (male: female) & 28:19 & 18:15 & 0.6540 \\
\hline Age (years) & $71.957 \pm 4.777$ & $72.848 \pm 3.392$ & $0.662 \Delta$ \\
\hline Education levels (years) & $15.894 \pm 11.429$ & $14.742 \pm 2.889$ & $0.124 \Delta$ \\
\hline Clinical dementia rating (CDR) & 0.5 & 0 & - \\
\hline Mini mental state exam (MMSE) & $26.979 \pm 1.525$ & $28.182 \pm 1.334$ & $0.001 * \Delta$ \\
\hline Auditory verbal learning test delayed recall & $2.894 \pm 1.747$ & $7.970 \pm 1.845$ & $0.000^{*} \Delta$ \\
\hline Rey-Osterrieth complex figure test delayed recall & $10.787 \pm 7.395$ & $16.578 \pm 7.009$ & $0.002^{*} \Delta$ \\
\hline Trail making test A (seconds) & $94.404 \pm 34.518$ & $73.818 \pm 29.591$ & $0.006^{*} \Delta$ \\
\hline Trail making test B (seconds) & $189.447 \pm 77.565$ & $139.849 \pm 43.953$ & $0.003^{*} \Delta$ \\
\hline Symbol digit modalities test & $26.575 \pm 9.930$ & $34.000 \pm 10.161$ & $0.003^{*} \Delta$ \\
\hline Clock drawing test & $8.192 \pm 1.676$ & $9.000 \pm 1.047$ & $0.011^{* \Delta}$ \\
\hline Digit span test & $12.106 \pm 2.119$ & $13.091 \pm 2.127$ & $0.027^{* \Delta}$ \\
\hline
\end{tabular}

Note: data are presented as mean $\pm \mathrm{SD}$. *indicates had statistical difference between groups, $P$ value $<0.05$. $\bigcirc$ : The $P$ value was obtained by Pearson chi-square test. $\triangle$ : The $P$ value was obtained by Mann-Whitney $U$ test, which was used here due to the data not being normally distributed.

span test), and executive functions (trail making test B). Further comparisons of the neuropsychological battery between the three genotypes of aMCI patients showed no significant differences, all $P>0.05$ (Table 2).

\section{The influence of diseases $\times$ genotypes on ALFF}

(1) Brain regions showing a significant main effect of groups were identified in the frontal cortex (left inferior gyrus), temporal cortex (left superior and middle lobes), parietal cortex ((right precuneus (Pcu)), occipital cortex (right calcarine) and cerebellar cortex (right posterior lobe), while main effects of genotypes were found only in the frontal cortex (bilateral middle, left inferior and right superior gyri, right anterior cingulum). In particular, regions associated with an interaction of groups $\times$ genotypes were found in the parietal cortex (left Pcu), frontal cortex (left superior, middle, and medial gyri, right anterior cingulum), left middle occipital lobe and the bilateral posterior lobes of the cerebellum (for details see Table 3 and Figure 1).

\section{The influence of factors on changes in ALFF}

Post hoc test: a) Compared to the control group, the aMCI group showed decreased ALFF in regions of the dominant hemisphere, including in the left inferior frontal gyrus and the left superior and middle temporal lobe, while increased ALFF was shown in the right Pcu and calcarine regions (Figure 2A). b) Compared to the CT genotype, the CC genotype showed increased ALFF in the left middle and inferior frontal gyri, and compared to the TT genotype, the CC genotype also showed increased ALFF in right medial and superior frontal gyri and the right anterior cingulum, while there were no significant differences between the $\mathrm{CT}$ and TT genotypes (Figure 2B). c) Further analysis of the interactions between groups and genotypes revealed that in both the aMCI and control groups, the CC genotype showed significantly higher ALFF than the CT and TT genotypes. In addition, we found that the ALFF value for each genotype in the control group was higher than that of the aMCI group, based on visual comparisons (no significant difference in statistics) (Figure 2C).

Table 2 Neuropsychological data between three genotype groups in aMCl patients

\begin{tabular}{llllll}
\hline Items & $\mathbf{C C}(\mathbf{n}=\mathbf{1 3})$ & $\mathbf{C T}(\mathbf{n}=\mathbf{2 3})$ & $\mathbf{T T}(\mathbf{n}=\mathbf{1 1})$ & Chi-square & $\mathbf{P}$ \\
\hline Mini-mental state exam (MMSE) & & 22.62 & 27.48 & 18.36 & 3.714 \\
\hline Auditory verbal learning test delayed recall & 26.00 & 21.33 & 27.23 & 1.863 \\
\hline Rey-Osterrieth complex figure test delayed recall & 26.58 & 21.74 & 25.68 & 1.254 & .394 \\
\hline Trail making test A (seconds) & 23.46 & 21.78 & 29.27 & 2.250 \\
\hline Trail making test B (seconds) & 20.15 & 24.85 & 26.77 & 1.561 \\
\hline Symbol digit modalities test & 24.00 & 25.48 & 20.91 & .325 \\
\hline Clock drawing test & 20.50 & 26.43 & 23.05 & .429 & 1.747 \\
\hline Digit span test & 27.88 & 25.11 & 17.09 & .661 \\
\hline
\end{tabular}

Note: The $\mathrm{P}$ value was obtained by Kruskal-Wallis test (a type of nonparametric test) as the statistical test, as the data of the three groups were not normally distributed. 
Table 3 Groups $\times$ genotypes ANOVA of ALFF

\begin{tabular}{|c|c|c|c|c|c|c|}
\hline \multirow[t]{2}{*}{ Brain region } & \multirow[t]{2}{*}{ BA } & \multicolumn{3}{|c|}{ Peak MNI coordinates (mm) } & \multirow[t]{2}{*}{ Peak $F$ value } & \multirow[t]{2}{*}{ Cluster size } \\
\hline & & $\mathbf{x}$ & y & $\mathbf{z}$ & & \\
\hline \multicolumn{7}{|l|}{ (1) Main effect of groups } \\
\hline L inferior frontal gyrus/superior temporal lobe/middle temporal lobe & 22/38/45 & -57 & 21 & 21 & 26.78 & 23841 \\
\hline R calcarine/precuneus/cerebellum posterior lobe & $7 / 18$ & 6 & -63 & -18 & 12.27 & 15120 \\
\hline \multicolumn{7}{|l|}{ (2) Main effect of genotypes } \\
\hline L middle frontal gyrus/inferior frontal gyrus & $9 / 45$ & -57 & 21 & 18 & 19.53 & 13851 \\
\hline R medial frontal gyrus/superior frontal gyrus/ anterior cingulum & 10 & 6 & 63 & 6 & 12.88 & 13608 \\
\hline \multicolumn{7}{|l|}{ (3) Groups $\times$ genotypes interaction } \\
\hline $\begin{array}{l}\text { L superior frontal gyrus/middle frontal gyrus/ medial frontal gyrus/R } \\
\text { anterior cingulum }\end{array}$ & $9 / 32 / 46$ & -54 & 27 & 21 & 13.14 & 19278 \\
\hline L middle occipital lobe/cuneus/precuneus & 19 & -21 & -96 & 24 & 9.34 & 10557 \\
\hline B cerebellum posterior lobe & - & -24 & -60 & -24 & 8.56 & 18468 \\
\hline
\end{tabular}

Note: a corrected threshold by Monte Carlo stimulation at $P<0.05$. Cluster size is in mm3. BA, Brodmann's area; B, bilateral; L, left; MNI. Montreal Neurological Institute; R, right.

Correlations between ALFF values and behavioral scores Correlations between the ALFF values of the clusters showing significant interactions with the neuropsychological tests scores are shown in Figure 3. There was a positive correlation in the left frontal cortex between ALFF and behavioral tests in the memory domain in the $\mathrm{CC}$ genotype, and a negative correlation in the right frontal cortex between ALFF and MMSE in the CT genotype, while in the TT genotype, we found a negative correlation in the bilateral frontal cortex and a positive correlation in the left posterior cerebellum between ALFF and the nonmemory domains.

\section{Discussion}

In this imaging genetics analysis, we investigated for the first time the effect of rs1143627 ( $-31 \mathrm{~T}>\mathrm{C}$ ) loci polymorphism of $I L 1 B$ gene on brain regional spontaneous activity in aged subjects presenting with aMCI and normally aging participants. We found that, among the $\mathrm{CC}$, CT, and TT genotypes, many brain regions showed significant differences in ALFF between the aMCI patients and the controls. Interestingly, we found that the regions of interaction between the genotypes and the groups showed some overlap with the default mode networks, including the $\mathrm{Pcu}$, the superior frontal gyrus, the anterior cingulum cortex (ACC) and some other frontal regions. Further behavioral significance suggested a role for the rs1143627 polymorphism in regional neuronal activity that has some effects on cognitive aging.

The protein IL-1 $\beta$, encoded by a $7.5 \mathrm{~kb}$ gene with seven exons, is regulated both by distal and proximal promoter elements [32]. The promoter SNP locus rs1143627 $(-31 \mathrm{~T}>\mathrm{C})$ is located in a TATA-box motif, which markedly affects DNA-protein interactions in vitro [33], and the change from $-31 \mathrm{~T}$ to $-31 \mathrm{C}$ may disrupt the TATA-box, leading to potential reduced or abolished transcriptional activity of the promoter $[34,35]$.A genome-wide association study (GWAS) confirmed that $-31 \mathrm{~T}>\mathrm{C}$ had the highest LOD score, providing strong, unbiased evidence that this SNP is functional and that IL-1 $\beta$ mRNA expression is a heritable trait [36].

It has been demonstrated both in vivo and in vitro that basal IL-1 $\beta$ expression is involved in the physiological long-term potentiation (LTP), a process believed to underlie certain forms of learning and memory [37]. At sufficient

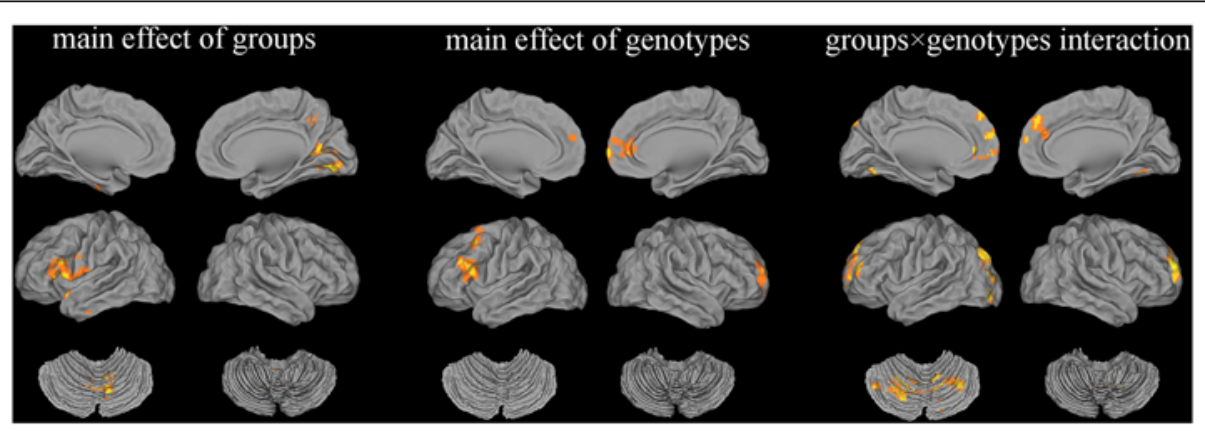

Figure 1 Groups $\times$ genotypes ANOVA of ALFF. Thresholds were set at a corrected $P<0.05$, determined by Monte Carlo stimulation. 


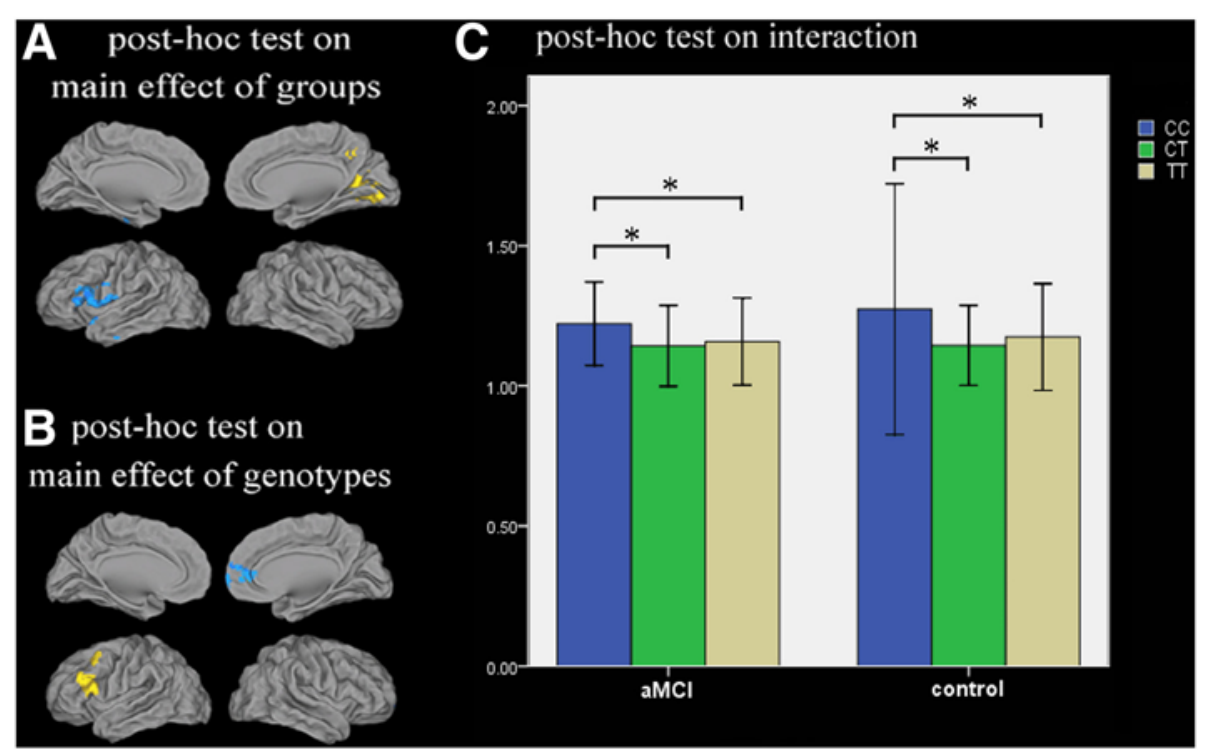

Figure 2 Post hoc test. Thresholds were set at a corrected $P<0.05$, determined by Monte Carlo stimulation. (A) Compared with controls group, aMCI group showed increased ALFF in right Pcu/calcarine, while decreased ALFF in left inferior frontal gyrus and superior/middle temporal lobe (yellow: aMCI >control, blue: aMCl <control). (B) Compared with CT genotype, CC genotype showed increased ALFF in left middle/inferior frontal gyrus, and compared with $\Pi$ genotype, CC genotype also showed increased ALFF in right medial/superior frontal gyrus and right anterior cingulum, while there were no significance between $\mathrm{CT}$ and $T$ T genotype (yellow: $C C>C T$, blue: $C C>T T$ ). (C) Further analysis of interaction between groups and genotypes found that both in aMCl and control groups, CC genotype showed significant higher ALFF than CT/TT genotype. The ALFF value for each genotype in controls group was higher than that in aMCl group based on visual comparisons (no significant difference in statistics). ${ }^{*} P<0.05$.

concentrations, IL-1 $\beta$ has an inhibitory effect on LTP in many regions of the hippocampus [38]. Additionally, the injection of lipopolysaccharide (LPS), a potent inducer of IL-1 $\beta$ expression, into the CA1 regions of the rat hippocampus results in learning and memory deficits [39]. Postmortem brain tissue from patients with early-stage AD showed significantly increased expression of caspase-1 (CASP1), also known as IL-1 $\beta$ converting enzyme (ICE) [40]. A previous meta-analysis of cytokines found a significantly higher concentration of $\mathrm{IL}-1 \beta$ in the peripheral blood of AD patients [6]. A case-control study on CSF concluded that intrathecal inflammation precedes the development of $\mathrm{AD}$ [41], and found the levels of IL-1 $\beta$ were significantly correlated with the levels of tau and $A \beta$ in patients with $\mathrm{MCI}$ who had progressed to $\mathrm{AD}$ at the follow-up nine months later. Furthermore, it was found the IL-1 $\beta$ levels were correlated to MMSE at both baseline and follow-up assessments. There have been many associating IL1B gene SNPs with the risk of occurrence of AD, but the results are controversial (see http://www.alzgene. org/). A previous genetic association study of Chinese participants did not detect any genotypic or allelic frequency differences in $I L 1 B-31 C / T$ between an $\mathrm{AD}$ group and a control group, and concluded that the $-31 \mathrm{C} / \mathrm{T}$ polymorphism was not a risk factor for $\mathrm{AD}$ [42], while the result needs to be further confirmed.

Our study showed decreased ALFF in the inferior frontal gyrus, the superior temporal lobe and the middle

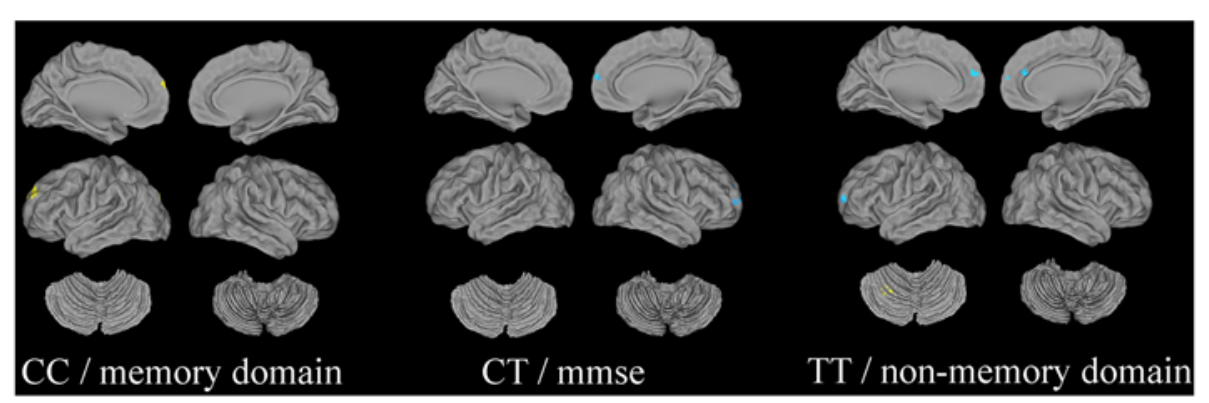

Figure 3 Correlations between ALFF and neuropsychological tests. Thresholds were set at a corrected $P<0.05$, determined by Monte Carlo stimulation (yellow: positive correlation; blue: negative correlation). 
temporal lobe in aMCI patients, which was consistent with previous hypoperfusion shown by SPECT and hypometabolism shown by PET in aMCI/AD [43,44]. It is worth noting that the frontal and temporal lobes, together with the parietal and occipital cortices, were previously identified with more accumulation of ${ }^{11} \mathrm{C}$ Pittsburgh compound B (PIB), consistent with brain regions containing $A \beta$ deposits [45]. A previous resting state fMRI study also found that reductions in regional activation [46,47], regional homogeneity $[10,48]$ and functional connectivity [49-52] were associated with both $\mathrm{AD}$ and $\mathrm{MCI}$ patients. A recent combined structural and resting-state fMRI study [53] found broad frontotemporal grey matter loss in $\mathrm{MCI}$ patients by voxel-based morphometry (VBM) analysis, and found regions of decreased connectivity to the posterior cingulated cortex (PCC), which provides further support for our findings. In the current study, we also observed increased ALFF in the aMCI group, including in the occipital cortex (calcarine), parietal cortex (Pcu) and cerebellar cortex. Jia and colleagues [19] found increased ALFF/fractional ALFF (fALFF) activity in aMCI patients in several occipital regions, which is consistent with the present study. A previous PET study [54] showed a significant increase in metabolism in the occipital cortex in aMCI brains. Researchers have also observed that baseline aMCI patients have increased cerebellar ALFF compared to controls [55]. Thus, our results of ALFF in aMCI suggested that aMCI patients might have relatively conserved spontaneous brain activity in these regions. The increased ALFF in the Pcu of aMCI patients in this study was in contrast to previous studies, which showed a decrease in ALFF. A structural MRI study showed that Pcu undergoes significant atrophy in MCI and mild AD [56]. In addition, lower functional connectivity within the default mode network (DMN) in the Pcu and PCC was detected in $\mathrm{AD}$ patients by an independent component analysis (ICA) [57]. The PCC and Pcu of aMCI patients displayed decreased ALFF/fALFF in a recent study [19]. We found similar results regarding Pcu in the present study. A recent resting state fMRI study [58] identified PCC and Pcu hyperfunctional connectivity at baseline in aMCI subjects, and a substantial decrease in these connections was evident at follow-up, compared to matched controls. Additionally, Jones et al. [59] analyzed task-free fMRI data with both ICA and seed-based analysis, identifying the posterior DMN as having a decline in connectivity, while an increase in connectivity was observed in the Pcu when atrophy correction was applied. A previous study [60] demonstrated that endogenous neuronal activity regulated the regional concentration of interstitial fluid $A \beta$ levels, which drive local $A \beta$ aggregation. It is uncertain whether some regions with early increased regional neural activity and hyperfunctional connectivity could be a mechanism of compensation for $\mathrm{AD}$ pathology or represent a more proximate event leading to cognitive dysfunction. In summary, our results suggested that aMCI patients had abnormal ALFF in their intrinsic brain activity.

In this study, we observed that the rs1143627 $\mathrm{C} / \mathrm{T}$ genotype had main effects on the frontal cortex, including the superior, middle, inferior and medial frontal gyri, and the ACC. SNPs in the ILIB gene were shown to have effects on the responsiveness of the amygdala and ACC to emotional stimulation in major depression [61]. Additional brain regions that had interactions with groups were in the frontal, parietal and occipital cortices and in the posterior lobe of the cerebellum. A recent genetic neuroimaging study [62] detected the effect of the $I L 1 B$ gene on frontal cortex function in schizophrenia. A mammalian genome study identified $I L 1 B$ gene polymorphisms that modulated scrapie (a type of neurodegenerative disease) susceptibility in sheep and goats, and found IL-1 $\beta$ expression in the cerebellum [63]. These studies supported our findings of the genetic effects of rs1143627 on different brain regions. In addition, the $\mathrm{CC}$ genotype showed significantly higher ALFF values than the CT and TT genotypes in both aMCI and control participants, and each genotype showed different or completely opposite results in the association between ALFF and cognitive scores. This study did not find the frequency of the three genotypes between groups, consistent with a previously mentioned study [42]. We did not find any significant effects of the genotypes on cognition in aMCI patients. This was not in contradiction to the behavioral significance, as the pathophysiological process of $\mathrm{AD}$ is thought to begin many years, even decades, before the onset of clinical dementia [64,65]. Thus, further large-scale association replicated and longitudinal studies are needed.

There were biological and technical limitations to this study that must be acknowledged. First, there was biological and clinical heterogeneity in the sample of aMCI participants as recruitment was based only on clinical criteria. Some participants may not display AD pathology, contaminating the sample with non-AD cases. The heterogeneity could be minimized through a combination of CSF and PET biomarkers. Second, as a result of the limitation of the sample size, further adjustment for multiple testing outside the domain of imaging will be necessary and replication in independent samples is also required to further establish the gene-imaging phenotype association. Third, previous resting state fMRI studies $[66,67]$ have demonstrated very moderate test-retest reliability based on Monte Carlo simulations and, because calculation methods will be improved with time, new analysis methods could be applied to future studies. 


\section{Conclusions}

In this study, we provide evidence that aMCI patients had abnormal ALFF in many brain regions, including in the Pcu, the calcarine, the inferior frontal gyrus and the superior and middle temporal lobe. In particular, we showed that the rs1143627 $\mathrm{C} / \mathrm{T}$ polymorphism might have an effect on spontaneous regional brain activity in aMCI patients.

\section{Abbreviations}

ACC: Anterior cingulum cortex; AD: Alzheimer's disease; ADHD: Attention deficit hyperactivity disorder; ADL: Activities of daily living; ALFF: Amplitude of low-frequency fluctuation; aMCl: amnestic mild cognitive impairment; $A B$ : amyloid beta; AVLT: Auditory verbal learning test; BOLD: Blood oxygenation level-dependent; CASP1: Caspase-1; CDR: Clinical dementia rating scale; DNM: Default mode network; fALFF: fractional ALFF; fMRI: Functional magnetic resonance imaging; FWHM: Full width at half maximum; GWAS: Genome-wide association study; ICA: Independent component analysis; ICE: IL-1 $\beta$ converting enzyme; IL-1 $\beta$ : Interleukin-1 beta; LPS: Lipopolysaccharide; LTP: Long-term potentiation; MCI: Mild cognitive impairment; MMSE: Mini-mental state examination; MS: Multiple sclerosis; NINCDS-ADRDA: National Institute of Neurological and Communicative Disorders and Stroke and the Alzheimer's Disease and Related Disorders Association; PCC: Posterior cingulated cortex; Pcu: Precuneus; PET: Positron emission tomography; PIB: Pittsburgh compound B; PTSD: Posttraumatic stress disorder; SNP: Single nucleotide polymorphism; SPECT: Single-photon emission computed tomography; SPGR: Spoiled gradient echo; VBM: Voxelbased morphometry.

\section{Competing interests}

The authors declare that they have no competing interests.

\section{Authors' information}

Zhijun Zhang is a professor of Southeast University, the person in charge of the Department of Neurology, affiliated ZhongDa Hospital of Southeast University and the Institute of Neuropsychiatry of Southeast University. She has been doing research on amnestic mild cognitive impairment, both on gene and brain imaging studies, for more than seven years, and her papers have been published by journals such as Biological Psychiatry, the Lancet and so on.

\section{Authors' contributions}

Liying Zhuang analyzed the data, made figures and tables and wrote the manuscript. Xiaoyan Liu, Xiaohui Xu, and Hao Shu helped analyze the data. Chunxian Yue designed the study. Feng Bai helped analyze the data and revise the manuscript. Hui Yu and Yongmei Shi contributed to the study conception and design and acquisition of data. Zhijun Zhang contributed to the study conception and design and manuscript revision. All authors provided editorial assistance and have read and approved the final version of the manuscript.

\section{Acknowledgements}

This research was partly supported by the National Natural Science Foundation of China (Numbers 30825014 ZZ, 81171021 YS).

Received: 19 March 2012 Accepted: 8 November 2012 Published: 1 December 2012

\section{References}

1. Petersen RC, Morris JC: Mild cognitive impairment as a clinical entity and treatment target. Arch Neurol 2005, 62:1160-1163. discussion 1167.

2. Petersen RC, Doody R, Kurz A, Mohs RC, Morris JC, Rabins PV, Ritchie K, Rossor M, Thal L, Winblad B: Current concepts in mild cognitive impairment. Arch Neurol 2001, 58:1985-1992.

3. Jack CJ, Knopman DS, Jagust WJ, Shaw LM, Aisen PS, Weiner MW, Petersen RC, Trojanowski JQ: Hypothetical model of dynamic biomarkers of the Alzheimer's pathological cascade. Lancet Neurol 2010, 9:119-128.
4. Flood DG, Marek GJ, Williams M: Developing predictive CSF biomarkers - a challenge critical to success in Alzheimer's disease and neuropsychiatric translational medicine. Biochem Pharmacol 2011, 81:1422-1434.

5. Akiyama H, Barger S, Barnum S, Bradt B, Bauer J, Cole GM, Cooper NR, Eikelenboom P, Emmerling M, Fiebich BL, Finch CE, Frautschy S, Griffin WS, Hampel H, Hull M, Landreth G, Lue L, Mrak R, Mackenzie IR, McGeer PL, O'Banion MK, Pachter J, Pasinetti G, Plata-Salaman C, Rogers J, Rydel R, Shen Y, Streit W, Strohmeyer R, Tooyoma I, et al: Inflammation and Alzheimer's disease. Neurobiol Aging 2000, 21:383-421.

6. Swardfager W, Lanctot K, Rothenburg L, Wong A, Cappell J, Herrmann N: A meta-analysis of cytokines in Alzheimer's disease. Biol Psychiatry 2010, 68:930-941.

7. Olson L, Humpel C: Growth factors and cytokines/chemokines as surrogate biomarkers in cerebrospinal fluid and blood for diagnosing Alzheimer's disease and mild cognitive impairment. Exp Gerontol 2010, 45:41-46.

8. Guasch JF, Bertina RM, Reitsma PH: Five novel intragenic dimorphisms in the human interleukin-1 genes combine to high informativity. Cytokine 1996, 8:598-602.

9. Biswal B, Yetkin FZ, Haughton VM, Hyde JS: Functional connectivity in the motor cortex of resting human brain using echo-planar MRI. Magn Reson Med 1995, 34:537-541.

10. He Y, Wang L, Zang Y, Tian L, Zhang X, Li K, Jiang T: Regional coherence changes in the early stages of Alzheimer's disease: a combined structural and resting-state functional MRI study. Neuroimage 2007, 35:488-500

11. Zhang Z, Lu G, Zhong Y, Tan Q, Chen H, Liao W, Tian L, Li Z, Shi J, Liu Y: fMRI study of mesial temporal lobe epilepsy using amplitude of low-frequency fluctuation analysis. Hum Brain Mapp 2010, 31:1851-1861

12. Hoptman MJ, Zuo XN, Butler PD, Javitt DC, D'Angelo D, Mauro CJ, Milham MP: Amplitude of low-frequency oscillations in schizophrenia: a resting state fMRI study. Schizophr Res 2010, 117:13-20.

13. Huang $X Q$, Lui $S$, Deng W, Chan $R C$, Wu QZ, Jiang LJ, Zhang JR, Jia ZY, Li XL, Li F, Chen L, Li T, Gong QY: Localization of cerebral functional deficits in treatment-naive, first-episode schizophrenia using resting-state fMRI. Neuroimage 2010, 49:2901-2906.

14. Jiang GH, Qiu YW, Zhang XL, Han LJ, Lv XF, Li LM, Lin CL, Zhuo FZ, Hu SY, Tian JZ: Amplitude low-frequency oscillation abnormalities in the heroin users: a resting state fMRI study. Neuroimage 2011, 57:149-154.

15. Yin Y, Li L, Jin C, Hu X, Duan L, Eyler LT, Gong Q, Song M, Jiang T, Liao M, Zhang Y, Li W: Abnormal baseline brain activity in posttraumatic stress disorder: a resting-state functional magnetic resonance imaging study. Neurosci Lett 2011, 498:185-189.

16. Yang H, Wu QZ, Guo LT, Li QQ, Long XY, Huang XQ, Chan RC, Gong QY: Abnormal spontaneous brain activity in medication-naive ADHD children: a resting state fMRI study. Neurosci Lett 2011, 502:89-93.

17. Zang YF, He Y, Zhu CZ, Cao QJ, Sui MQ, Liang M, Tian LX, Jiang TZ, Wang YF: Altered baseline brain activity in children with ADHD revealed by resting-state functional MRI. Brain Dev 2007, 29:83-91.

18. Lowe MJ, Phillips MD, Lurito JT, Mattson D, Dzemidzic M, Mathews VP: Multiple sclerosis: low-frequency temporal blood oxygen level-dependent fluctuations indicate reduced functional connectivity initial results. Radiology 2002, 224:184-192.

19. Han Y, Wang J, Zhao Z, Min B, Lu J, Li K, He Y, Jia J: Frequency-dependent changes in the amplitude of low-frequency fluctuations in amnestic mild cognitive impairment: a resting-state fMRI study. Neuroimage 2011, 55:287-295.

20. Petersen RC, Smith GE, Waring SC, Ivnik RJ, Tangalos EG, Kokmen E: Mild cognitive impairment: clinical characterization and outcome. Arch Neurol 1999, 56:303-308.

21. Winblad B, Palmer K, Kivipelto M, Jelic V, Fratiglioni L, Wahlund LO, Nordberg A, Backman L, Albert M, Almkvist O, Arai H, Basun H, Blennow K, de Leon M, DeCarli C, Erkinjuntti T, Giacobini E, Graff C, Hardy J, Jack C, Jorm A, Ritchie K, van Duijn C, Visser P, Petersen RC: Mild cognitive impairment-beyond controversies, towards a consensus: report of the International Working Group on Mild Cognitive Impairment. J Intern Med 2004, 256:240-246.

22. Lowe MJ, Mock BJ, Sorenson JA: Functional connectivity in single and multislice echoplanar imaging using resting-state fluctuations. Neuroimage 1998, 7:119-132. 
23. Yang $H$, Long $X Y$, Yang $Y$, Yan $H$, Zhu CZ, Zhou XP, Zang YF, Gong QY: Amplitude of low frequency fluctuation within visual areas revealed by resting-state functional MRI. Neuroimage 2007, 36:144-152.

24. Lui S, Huang X, Chen L, Tang H, Zhang T, Li X, Li D, Kuang W, Chan RC, Mechelli A, Sweeney JA, Gong Q: High-field MRI reveals an acute impact on brain function in survivors of the magnitude 8.0 earthquake in China. Proc Natl Acad Sci USA 2009, 106:15412-15417.

25. Long $X Y$, Zuo $X N$, Kiviniemi $V$, Yang $Y$, Zou QH, Zhu CZ, Jiang TZ, Yang $H_{\text {, }}$ Gong QY, Wang L, Li KC, Xie S, Zang YF: Default mode network as revealed with multiple methods for resting-state functional MRI analysis. J Neurosci Methods 2008, 171:349-355.

26. Zou QH, Zhu CZ, Yang Y, Zuo XN, Long XY, Cao QJ, Wang YF, Zang YF: An improved approach to detection of amplitude of low-frequency fluctuation (ALFF) for resting-state fMRI: fractional ALFF. J Neurosci Methods 2008, 172:137-141.

27. Yan C, Liu D, He Y, Zou Q, Zhu C, Zuo X, Long X, Zang Y: Spontaneous brain activity in the default mode network is sensitive to different resting-state conditions with limited cognitive load. PLoS One 2009, 4:e5743.

28. Oakes TR, Fox AS, Johnstone T, Chung MK, Kalin N, Davidson RJ: Integrating VBM into the general linear model with voxelwise anatomical covariates. Neuroimage 2007, 34:500-508.

29. Ashburner J, Friston KJ: Voxel-based morphometry - the methods. Neuroimage 2000, 11:805-821.

30. Good CD, Johnsrude IS, Ashburner J, Henson RN, Friston KJ, Frackowiak RS: A voxel-based morphometric study of ageing in 465 normal adult human brains. Neuroimage 2001, 14:21-36.

31. Jenkinson M, Bannister P, Brady M, Smith S: Improved optimization for the robust and accurate linear registration and motion correction of brain images. Neuroimage 2002, 17:825-841.

32. Shirakawa F, Saito K, Bonagura CA, Galson DL, Fenton MJ, Webb AC, Auron $P E$ : The human prointerleukin 1 beta gene requires DNA sequences both proximal and distal to the transcription start site for tissue-specific induction. Mol Cell Biol 1993, 13:1332-1344.

33. El-Omar EM, Carrington M, Chow WH, McColl KE, Bream JH, Young HA, Herrera J, Lissowska J, Yuan CC, Rothman N, Lanyon G, Martin M, Fraumeni JF Jr, Rabkin CS: Interleukin-1 polymorphisms associated with increased risk of gastric cancer. Nature 2000, 404:398-402.

34. Wobbe CR, Struhl K: Yeast and human TATA-binding proteins have nearly identical DNA sequence requirements for transcription in vitro. Mol Cell Biol 1990, 10:3859-3867.

35. Lind $H$, Haugen A, Zienolddiny S: Differential binding of proteins to the IL1B-31 T/C polymorphism in lung epithelial cells. Cytokine 2007, 38:43-48.

36. Dixon AL, Liang L, Moffatt MF, Chen W, Heath S, Wong KC, Taylor J, Burnett E, Gut I, Farrall M, Lathrop GM, Abecasis GR, Cookson WO: A genome-wide association study of global gene expression. Nat Genet 2007, 39:1202-1207

37. Schneider H, Pitossi F, Balschun D, Wagner A, Del RA, Besedovsky HO: A neuromodulatory role of interleukin-1 beta in the hippocampus. Proc Natl Acad Sci USA 1998, 95:7778-7783.

38. O'Connor JJ, Coogan AN: Actions of the pro-inflammatory cytokine IL-1 beta on central synaptic transmission. Exp Physiol 1999,

84:601-614.

39. Tanaka S, Ide M, Shibutani T, Ohtaki H, Numazawa S, Shioda S, Yoshida T: Lipopolysaccharide-induced microglial activation induces learning and memory deficits without neuronal cell death in rats. J Neurosci Res 2006, 83:557-566

40. Pompl PN, Yemul S, Xiang Z, Ho L, Haroutunian V, Purohit D, Mohs R, Pasinetti GM: Caspase gene expression in the brain as a function of the clinical progression of Alzheimer disease. Arch Neurol 2003, 60:369-376.

41. Tarkowski E, Andreasen N, Tarkowski A, Blennow K: Intrathecal inflammation precedes development of Alzheimer's disease. I Neurol Neurosurg Psychiatry 2003, 74:1200-1205.

42. Ma SL, Tang NL, Lam LC, Chiu HF: Lack of association of the interleukin-1 beta gene polymorphism with Alzheimer's disease in a Chinese population. Dement Geriatr Cogn Disord 2003, 16:265-268.

43. Minoshima S, Giordani B, Berent S, Frey KA, Foster NL, Kuhl DE: Metabolic reduction in the posterior cingulate cortex in very early Alzheimer's disease. Ann Neurol 1997, 42:85-94
44. Hirao K, Ohnishi T, Hirata Y, Yamashita F, Mori T, Moriguchi Y, Matsuda H, Nemoto K, Imabayashi E, Yamada M, Iwamoto T, Arima K, Asada T: The prediction of rapid conversion to Alzheimer's disease in mild cognitive impairment using regional cerebral blood flow SPECT. Neuroimage 2005 28:1014-1021.

45. Weaver JD, Espinoza R, Weintraub NT: The utility of PET brain imaging in the initial evaluation of dementia. J Am Med Dir Assoc 2007, 8:150-157.

46. Lustig C, Snyder AZ, Bhakta M, O'Brien KC, McAvoy M, Raichle ME, Morris JC, Buckner RL: Functional deactivations: change with age and dementia of the Alzheimer type. Proc Natl Acad Sci USA 2003, 100:14504-14509.

47. Rombouts SA, Barkhof F, Goekoop R, Stam CJ, Scheltens P: Altered resting state networks in mild cognitive impairment and mild Alzheimer's disease: an fMRI study. Hum Brain Mapp 2005, 26:231-239.

48. Bai F, Zhang Z, Yu H, Shi Y, Yuan Y, Zhu W, Zhang X, Qian Y: Default-mode network activity distinguishes amnestic type mild cognitive impairment from healthy aging: a combined structural and resting-state functional MRI study. Neurosci Lett 2008, 438:111-115.

49. Greicius MD, Srivastava G, Reiss AL, Menon V: Default-mode network activity distinguishes Alzheimer's disease from healthy aging: evidence from functional MRI. Proc Natl Acad Sci USA 2004, 101:4637-4642.

50. Wang K, Liang M, Wang L, Tian L, Zhang X, Li K, Jiang T: Altered functional connectivity in early Alzheimer's disease: a resting-state fMRI study. Hum Brain Mapp 2007, 28:967-978.

51. Sorg C, Riedl V, Muhlau M, Calhoun VD, Eichele T, Laer L, Drzezga A, Forstl $H$, Kurz A, Zimmer C, Wohlschlager AM: Selective changes of resting-state networks in individuals at risk for Alzheimer's disease. Proc Natl Acad Sci USA 2007, 104:18760-18765.

52. Bai F, Watson DR, Yu H, Shi Y, Yuan Y, Zhang Z: Abnormal resting-state functional connectivity of posterior cingulate cortex in amnestic type mild cognitive impairment. Brain Res 2009, 1302:167-174.

53. Wang $Z$, Liang $P$, Jia $X$, Jin $G$, Song H, Han Y, Lu J, Li K: The baseline and longitudinal changes of PCC connectivity in mild cognitive impairment: a combined structure and resting-state fMRI study. PLoS One 2012, 7:e36838.

54. Truchot L, Costes N, Zimmer L, Laurent B, Le Bars D, Thomas-Anterion C, Mercier B, Hermier M, Vighetto A, Krolak-Salmon P: A distinct [18 F]MPPF PET profile in amnestic mild cognitive impairment compared to mild Alzheimer's disease. Neuroimage 2008, 40:1251-1256.

55. Bai F, Liao W, Watson DR, Shi Y, Yuan Y, Cohen AD, Xie C, Wang Y, Yue C, Teng $Y$, Wu D, Jia J, Zhang Z: Mapping the altered patterns of cerebellar resting-state function in longitudinal amnestic mild cognitive impairment patients. J Alzheimers Dis 2011, 23:87-99.

56. Apostolova LG, Steiner CA, Akopyan GG, Dutton RA, Hayashi KM, Toga AW, Cummings $\mathrm{J}$, Thompson PM: Three-dimensional gray matter atrophy mapping in mild cognitive impairment and mild Alzheimer disease. Arch Neurol 2007, 64:1489-1495.

57. Binnewijzend MA, Schoonheim MM, Sanz-Arigita E, Wink AM, van der Flier WM, Tolboom N, Adriaanse SM, Damoiseaux JS, Scheltens P, van Berckel BN, Barkhof F: Resting-state fMRI changes in Alzheimer's disease and mild cognitive impairment. Neurobiol Aging 2012, 33:2018-2028.

58. Bai F, Watson DR, Shi Y, Wang Y, Yue C, Yuhuan T, Wu D, Yuan Y, Zhang Z: Specifically progressive deficits of brain functional marker in amnestic type mild cognitive impairment. PLoS One 2011, 6:e24271.

59. Jones DT, Machulda MM, Vemuri P, McDade EM, Zeng G, Senjem ML, Gunter JL, Przybelski SA, Avula RT, Knopman DS, Boeve BF, Petersen RC, Jack $C R$ Jr: Age-related changes in the default mode network are more advanced in Alzheimer disease. Neurology 2011, 77:1524-1531.

60. Bero AW, Yan P, Roh JH, Cirrito JR, Stewart FR, Raichle ME, Lee JM, Holtzman DM: Neuronal activity regulates the regional vulnerability to amyloid-beta deposition. Nat Neurosci 2011, 14:750-756.

61. Baune BT, Dannlowski U, Domschke K, Janssen DG, Jordan MA, Ohrmann P, Bauer J, Biros E, Arolt V, Kugel H, Baxter AG, Suslow T: The interleukin 1 beta (IL1B) gene is associated with failure to achieve remission and impaired emotion processing in major depression. Biol Psychiatry 2010, 67:543-549.

62. Fatjo-Vilas M, Pomarol-Clotet E, Salvador R, Monte GC, Gomar JJ, Sarro S, Ortiz-Gil J, Aguirre C, Landin-Romero R, Guerrero-Pedraza A, Papiol S, Blanch J, McKenna PJ, Fananas L: Effect of the interleukin-1beta gene on dorsolateral prefrontal cortex function in schizophrenia: a genetic neuroimaging study. Biol Psychiatry 2012, 72:758-765. 
63. Marcos-Carcavilla A, Calvo JH, Gonzalez C, Moazami-Goudarzi K, Laurent P, Bertaud M, Hayes H, Beattie AE, Serrano C, Lyahyai J, Martín-Burriel I, Alves E, Zaragoza P, Badiola JJ, Serrano M: IL-1 family members as candidate genes modulating scrapie susceptibility in sheep: localization, partial characterization, and expression. Mamm Genome 2007, 18:53-63.

64. Sperling RA, Aisen PS, Beckett LA, Bennett DA, Craft S, Fagan AM, Iwatsubo T, Jack CJ, Kaye J, Montine TJ, Park DC, Reiman EM, Rowe CC, Siemers E, Stern Y, Yaffe K, Carrillo MC, Thies B, Morrison-Bogorad M, Wagster MV,

Phelps CH: Toward defining the preclinical stages of Alzheimer's disease: recommendations from the National Institute on Aging-Alzheimer's

Association workgroups on diagnostic guidelines for Alzheimer's disease. Alzheimers Dement 2011, 7:280-292.

65. Hebert LE, Scherr PA, Beckett LA, Albert MS, Pilgrim DM, Chown MJ, Funkenstein HH, Evans DA: Age-specific incidence of Alzheimer's disease in a community population. JAMA 1995, 273:1354-1359.

66. Shehzad Z, Kelly AM, Reiss PT, Gee DG, Gotimer K, Uddin LQ, Lee SH, Margulies DS, Roy AK, Biswal BB, Petkova E, Castellanos FX, Milham MP: The resting brain: unconstrained yet reliable. Cereb Cortex 2009, 19:2209-2229.

67. Zuo XN, Kelly C, Adelstein JS, Klein DF, Castellanos FX, Milham MP: Reliable intrinsic connectivity networks: test-retest evaluation using ICA and dual regression approach. Neuroimage 2010, 49:2163-2177.

doi:10.1186/1742-2094-9-263

Cite this article as: Zhuang et al:: Association of the interleukin 1 beta gene and brain spontaneous activity in amnestic mild cognitive impairment. Journal of Neuroinflammation 2012 9:263.

\section{Submit your next manuscript to BioMed Central and take full advantage of:}

- Convenient online submission

- Thorough peer review

- No space constraints or color figure charges

- Immediate publication on acceptance

- Inclusion in PubMed, CAS, Scopus and Google Scholar

- Research which is freely available for redistribution 